\title{
Sensing Chemical Warfare Agent Simulants via Photonic Crystals of the Morpho didius Butterfly
}

\author{
Joshua D. Kittle, ${ }^{*}$ B Benjamin P. Fisher, Anthony J. Esparza, Aimee M. Morey, and Scott T. Iacono \\ Department of Chemistry, United States Air Force Academy, 2355 Fairchild Drive, 80840 Colorado Springs, Colorado, United States \\ Supporting Information
}

ABSTRACT: The rapid and portable detection of trace chemical warfare agents (CWAs) remains a challenge for the international security and monitoring community. This work reports the first use of natural photonic crystals $(\mathrm{PhCs})$ as vapor sensors for CWA simulants. Dimethyl methylphosphonate, a nerve agent simulant, and dichloropentane, a mustard gas simulant, were successfully detected at the parts per million level by processing visible light reflected from the $\mathrm{PhC}$ inherent to the wing scales of the Morpho didius butterfly. Additionally, modeling of this natural system suggested several parameters for enhancing the sensitivity of a synthetic PhC toward CWA simulants, including materials selection, structure, and spacing of the $\mathrm{PhC}$, and partial functionalization of the

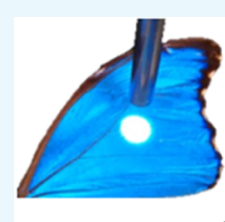
Signal Processing of Reflected Light

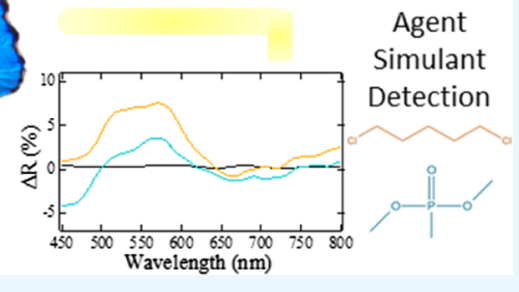
$\mathrm{PhC}$ toward the analyte of interest. Collectively, this study provides strategies for designing a sensitive, selective, rapid, and affordable means for CWA detection.

\section{INTRODUCTION}

The homeland defense and battlespace operation communities require portable and reliable sensors for chemical warfare agent (CWA) detection. The inherent toxicity of these organophosphates necessitates identification at the parts per trillion level and, to date, only expensive and nonportable instrumentation, such as mass spectrometry and nuclear magnetic resonance spectroscopy, have shown clear discrimination at the requisite sensitivity. ${ }^{1,2}$ However, a variety of other methods to detect CWA and CWA simulants have shown promise, such as colorimetric, ${ }^{3-6}$ chemiluminescence, ${ }^{7-14}$ porous silicon,,${ }^{15,16}$ nanomaterial, ${ }^{17-23}$ piezoelectric, ${ }^{24-27}$ and electrochemical techniques. ${ }^{28-30}$ These methods, though, are typically unsuitable for long-term field use owing to one or more challenges, such as inadequate sensitivity and/or selectivity, sensor poisoning, cost, robustness, or portability. ${ }^{31}$

Recent studies into both natural and synthetic photonic crystals (PhCs) have shown that these systems are sensitive to vapors with similar chemical and physical properties, offering a new means to rapidly identify and quantify trace analytes. ${ }^{32-34}$ $\mathrm{PhCs}$ rely on the principle of light interference when moving through periodic structures of varied refractive index and dielectric constant, resulting in photonic band gaps and structural color. ${ }^{35,36}$ Small changes in either the refractive index or the distance between layers of the periodic structure of the PhC can lead to significant changes in the wavelength of reflected light - the basis of sensing via PhCs. ${ }^{32}$ Sensing in the visible light spectrum requires periodicity of the layers in the nanometer range. Although three-dimensional $\mathrm{PhCs}$ at the nanometer scale have been fabricated, ${ }^{33}$ natural $\mathrm{PhCs}$ currently show a significantly higher sensitivity toward vapors because of their complex periodicity, as well as the polarity gradient found within the natural $\mathrm{PhC}$ architecture. ${ }^{34,37} \mathrm{~A}$ recent work has shown that the light reflected from the scales of the Morpho butterfly can be used to distinguish between vapors of similar polarity and refractive index..$^{37,38}$ The polarity gradient within the ridges and lamella of the scales led to aggregation of the vapor in certain regions, altering the dielectric properties of the $\mathrm{PhC}$ in a manner unique to each vapor analyzed and detectable at the parts per million level.

Despite the promise of PhCs as vapor sensors, few studies of CWA simulants and $\mathrm{PhCs}$ are found in the literature and are largely confined to photonic hydrogel sensors in the aqueous phase. Walker et al. pioneered the use of photonic hydrogel sensors by using polymerized crystalline colloidal arrays in conjunction with an enzyme chosen to swell or shrink the $\mathrm{PhC}$ periodicity in the presence of a CWA simulant. Concentrations of parathion and methyl parathion were detected at femtomolar and micromolar concentrations, respectively. ${ }^{6,39}$ Although this work has given rise to further studies of photonic hydrogels and CWA simulants, ${ }^{31}$ this synthetic, aqueous technique is not wellsuited to rapid, reversible, and cost-effective vapor detection of CWA simulants. Thus, although natural $\mathrm{PhC}$ sensors have shown selectivity to common vapors, such as methanol $(\mathrm{MeOH})$ and toluene, their selectivity and sensitivity toward CWA simulant vapors have not been established.

This work examines the viability of using a natural $\mathrm{PhC}$ and visible light reflectance to detect and discriminate between CWA simulant vapors and other common vapors. Wing scales of the Morpho didius butterfly, a well-characterized natural

Received: October 31, 2017

Accepted: November 10, 2017

Published: November 21, 2017 
$\mathrm{PhC},{ }^{40}$ were exposed to water, methanol $(\mathrm{MeOH})$, and ethanol $(\mathrm{EtOH})$, as well as the CWA simulants 1,5-dichloropentane (DCP, mustard gas simulant) and dimethyl methylphosphonate (DMMP, nerve agent simulant). Reflectance spectra in the presence of each vapor were collected at several concentrations, and the entire data set was processed using principal component analysis (PCA). Further, the reflectance spectra for each vapor were modeled by generating a periodic nanoarchitecture similar to that of the natural wing scale upon exposure to each vapor. Collectively, the results of this work are expected to provide the first baseline data set for natural $\mathrm{PhCs}$ as CWA simulant vapor sensors and suggest avenues for designing synthetic CWA simulant vapor sensors that outperform sensors based on natural systems.

\section{RESULTS AND DISCUSSION}

The nanostructure of the Morpho butterfly has been wellcharacterized in other studies..$^{32,34,37,38,43}$ In brief, Figure 1

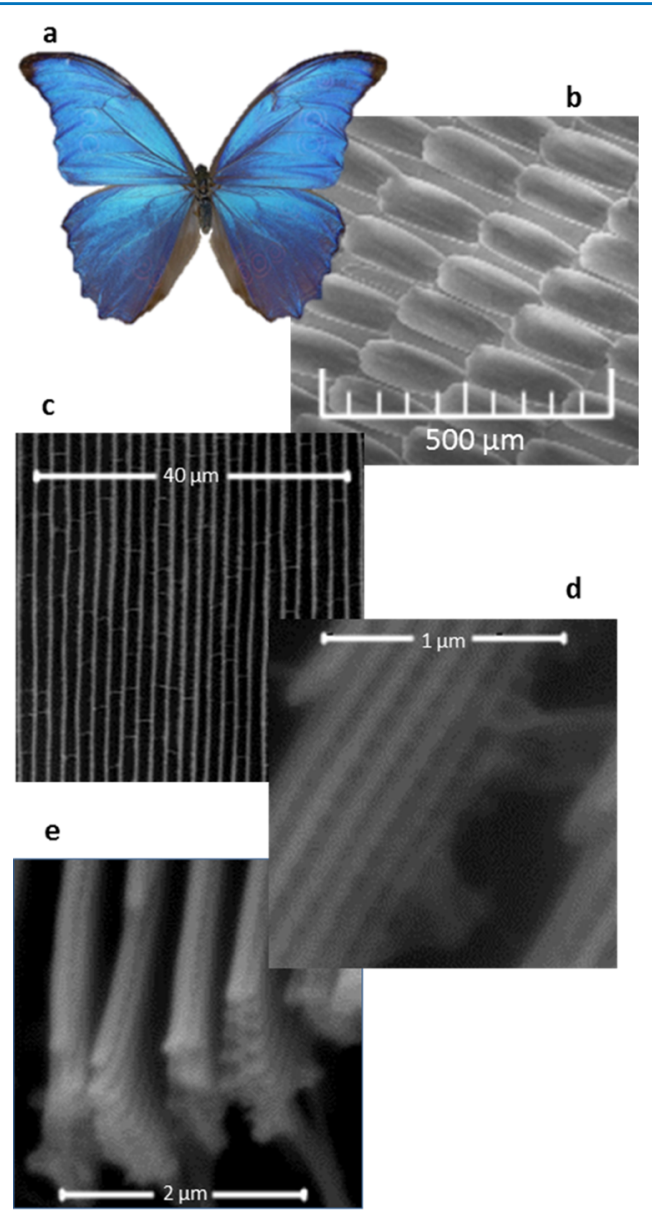

Figure 1. Morpho didius butterfly (a), M. didius cover and ground scales (b), ground scale ridges (c), ridges showing lamella and microribs (d), and ridge cross section showing lamella outcropping (e).

shows that wing scales are comprised of parallel ridges normal to the plane of the wing. A cross section of the wing shows that each ridge in turn contains a tapered, tree-like structure with seven lamellae extending on each side of the center of the ridge. Lamellae are roughly $70-80 \mathrm{~nm}$ thick with widths ranging from 200 to $400 \mathrm{~nm}$; the vertical spacing between lamella is on the order of $150 \mathrm{~nm}$, and the spacing between ridges is approximately $800 \mathrm{~nm}$. Hence, the wing scales are periodic on the nanometer scale, giving rise to photonic gaps in the visible light region. Unlike most synthetic $\mathrm{PhCs}$, studies have shown that a polarity gradient exists within the ridges of the wing scales, with higher polarities at the top of the ridges. This polarity gradient is the key to sensing via reflected light from the Morpho butterflies, as the location of the analyte within the structure also contributes to the wavelengths of the light reflected rather than refractive index alone. ${ }^{34,37}$ However, the sensitivity and selectivity of this natural system to CWA simulants had not been previously determined.

The sensitivity of the M. didius PhCs toward CWA simulants was studied by exposing the wing to a variety of analyte vapors, with the reflectance $(R)$ of each analyte measured individually at three different concentrations. As shown in Figure 2, a

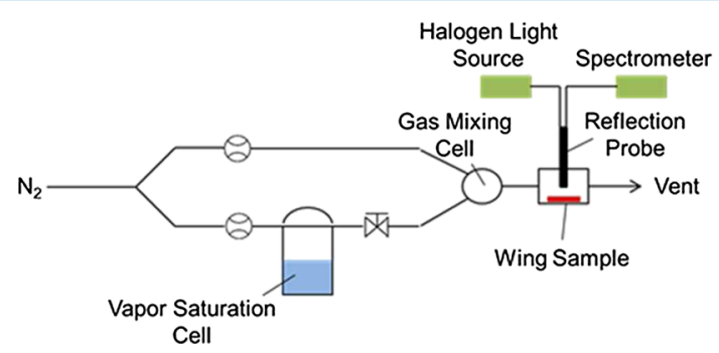

Figure 2. Schematic of the instrumentation for measuring reflectance spectra with varied analyte vapor concentrations.

manual system was used to vary the fraction of the analyte vapor in the nitrogen carrier gas, and the reflectance spectra were collected via fiber optics. The differential reflectance $(\Delta R)$ of DCP, DMMP, EtOH, MeOH, and water at each concentration relative to pure nitrogen is shown in Figure 3a-e. Raw reflectance data for all experimental runs can be found in the Supporting Information (Figure S1). Traces for each analyte are unique and vary in intensity with concentration, suggesting that the system is both selective and responsive to the vapors tested, including the CWA simulants. However, as evidenced by the smaller $\Delta R$ values relative to $\mathrm{EtOH}, \mathrm{MeOH}$, and water, the sensitivity of this system toward the CWA simulants is challenged by the relatively low saturated vapor pressures of DCP (2750 ppm) and DMMP (1575 ppm). ${ }^{44,45}$ Thus, the limits of detection toward these CWA simulants in this study are on the order of parts per million. Considering that the nerve agent VX is lethal at concentrations of $0.3 \mathrm{ppm},{ }^{31}$ this presents a challenge to use natural PhCs for CWA detection. However, as outlined below and in the literature, several avenues exist for increasing sensitivity via a synthetic mimic, including careful selection of $\mathrm{PhC}$ refractive index, targeted functionalization of certain regions within a $\mathrm{PhC}$ nanoarchitecture, and optimized $\mathrm{PhC}$ periodicity, among others. ${ }^{34}$ It is important to note that sensing nerve agents using an optimized, synthetic $\mathrm{PhC}$ is expected to provide significant advantages over current technologies in terms of sample acquisition and sensor portability, cost, and size. These advantages should enable the deployment of robust, real-time passive sensors and foster the integration of miniaturized sensors for rapid and remote forensic analysis.

To further examine the selectivity of the $M$. didius toward the CWA simulants, $\Delta R$ data for all analytes and concentrations were processed collectively using PCA. PCA is a commonly used multivariate analysis technique that reduces the dimensionality of a data set by determining the principle 

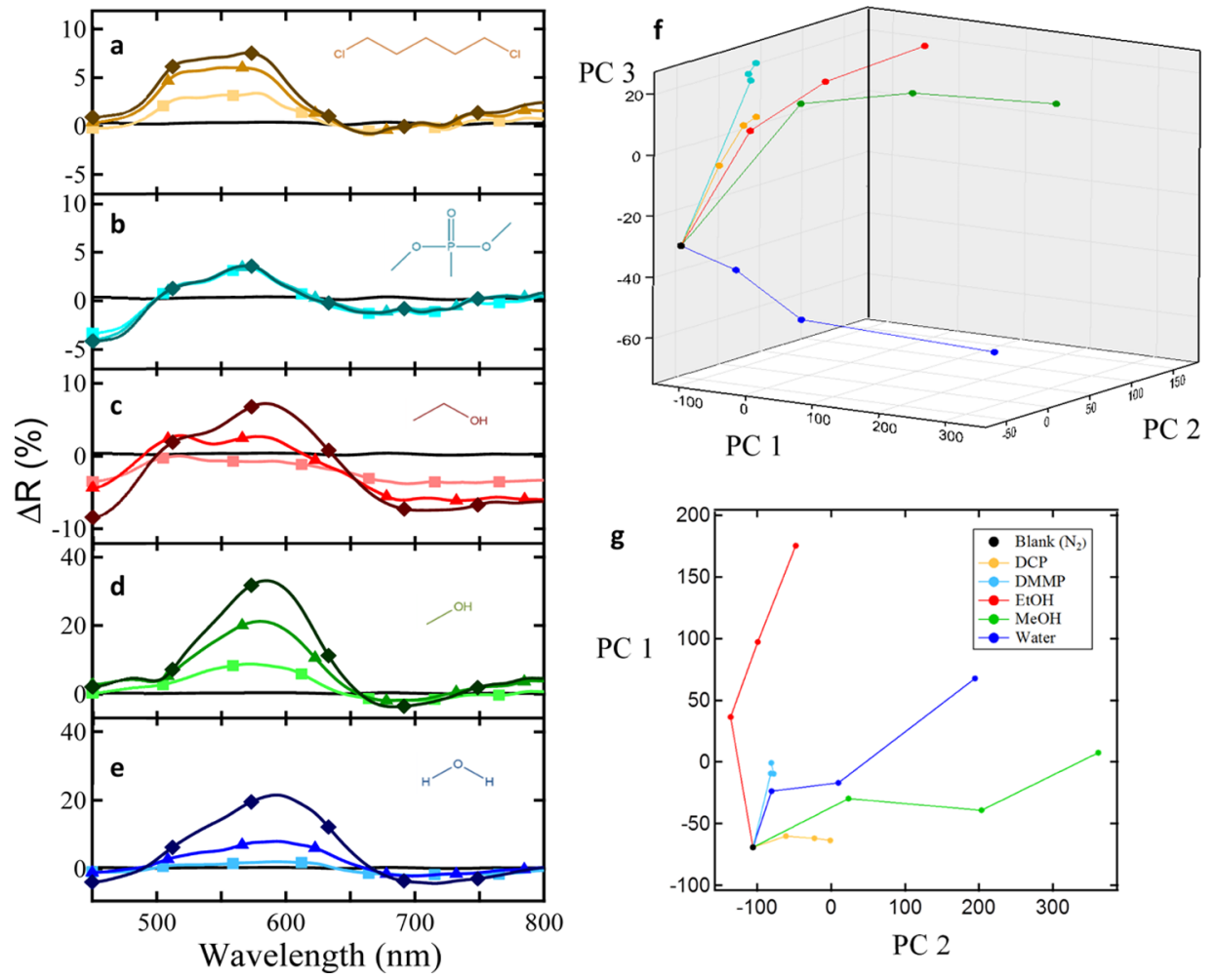

Figure 3. Differential reflectance spectra $(\Delta R)$ vs wavelength $(\mathrm{a}-\mathrm{e})$ at vapor concentrations of $0.15 P_{0}(\boldsymbol{\square}), 0.25 P_{0}(\boldsymbol{\Delta})$, and $0.50 P_{0}(\boldsymbol{\nabla})$ for $\mathrm{DCP}$ $(-)$, DMMP (-), EtOH $(-), \mathrm{MeOH}(-)$, and water $(-)$. Note that the $y$-axis scale differs for the CWA simulants. The PCA score plots (f,g) show discrimination of the vapors, where the first three principal components (PCs) captured $91.9 \%$ of the total spectral variance $(\mathrm{PC} 1=71.6 \%, \mathrm{PC} 2=$ $16.8 \%$, and PC $3=3.5 \%$ ).

components that capture the variance within that data. ${ }^{46}$ Twoand three-dimensional PCA score plots of the $\Delta R$ data for all five vapors are shown in Figure $3 \mathrm{f}, \mathrm{g}$, with data points indicating an increase in concentration from left to right. Similar to other studies of the light reflected from the Morpho butterfly upon exposure to $\mathrm{EtOH}, \mathrm{MeOH}$, and water, this work showed good analyte discrimination (e.g., distinct PCA curves), despite similar concentrations and refractive indices. ${ }^{38,43}$ Additionally, the data also indicate good selectivity toward the CWA simulants DCP and DMMP, despite their low vapor concentrations. Although PCA score plots of previous studies of the Morpho sulkowskyi indicated a trend with solvent polarity, ${ }^{37}$ the trend was not clearly observed for this study of the $M$. didius. As mentioned previously, the Morpho butterfly scales contain a polarity gradient moving up the lamella of the ridges, suggesting that analyte vapors aggregate within certain regions of the scale based on their polarity which significantly contributes to spectral differences. Studies indicated that removing the polarity gradient within the wing resulted in trends that matched the refractive index, similar to what is observed for traditional synthetic PhCs without a polarity gradient. $^{37}$ No clear trends in solvent polarity or refractive index was observed in this study, which highlights the complex, multivariate nature of vapor sensing via natural $\mathrm{PhC}$ systems. ${ }^{34}$ Regardless, the system studied here did show a good selectivity to CWA simulants, although the sensitivity toward these simulants must be improved for practical use as a vapor sensor for nerve agents.

Consequently, modeling was used to explore how a synthetic mimic of the M. didius $\mathrm{PhC}$ might be designed to improve the sensitivity toward CWA simulants (Figure 4). Although it is important to recall that sensing via natural $\mathrm{PhCs}$ is complex and interdependent on several variables, ${ }^{34}$ the simplified model presented here is of value in illustrating how various parameters might affect a synthetic $\mathrm{PhC}$ system. The model used in simulating the $\mathrm{PhC}$ reflectance was based on the structure of $M$. didius and is similar to that used by Jiang et al., with notable differences in the lamella and ridge spacing, as well as the consideration of vapor coating the ridges in addition to the lamella. ${ }^{43}$ Details of the model can be found in the Supporting Information (Figure S2). Reflectance data for all runs of the modeled $\mathrm{PhC}$ are provided in the Supporting Information (Figures S3-S7). Generally, the overall trace appearance and location of peak intensities with the wavelength of the model system are comparable to the experimental data, suggesting that the model $\mathrm{PhC}$ is a reasonable approximation of the natural system. Additionally, the generated reflectance spectra differ significantly from that obtained by Jiang et al., ${ }^{43}$ illustrating the significant effect that model parameters have on the generated spectra and the design precision required for $\mathrm{PhC}$ optimization.

Figure 4 demonstrates how three parameters-vapor concentration, vapor refractive index, and vapor location within the $\mathrm{PhC}$-affected the light reflected from the $\mathrm{PhC}$ model. As shown in Figure $4 a$, when the refractive index and the location of the analyte within the $\mathrm{PhC}$ are held constant, but concentration is increased, a slight red shift, as well as a peak intensity increase, is evident near 500 and $600 \mathrm{~nm}$. These phenomena resulted from the increase in refractive index of the $\mathrm{PhC}$ model caused by the addition of thicker layers of condensed analyte to the PhC surface. ${ }^{36}$ For Figure $4 \mathrm{~b}$, the concentration and the analyte location within the $\mathrm{PhC}$ are held constant, whereas the refractive index is varied. Of note, for 

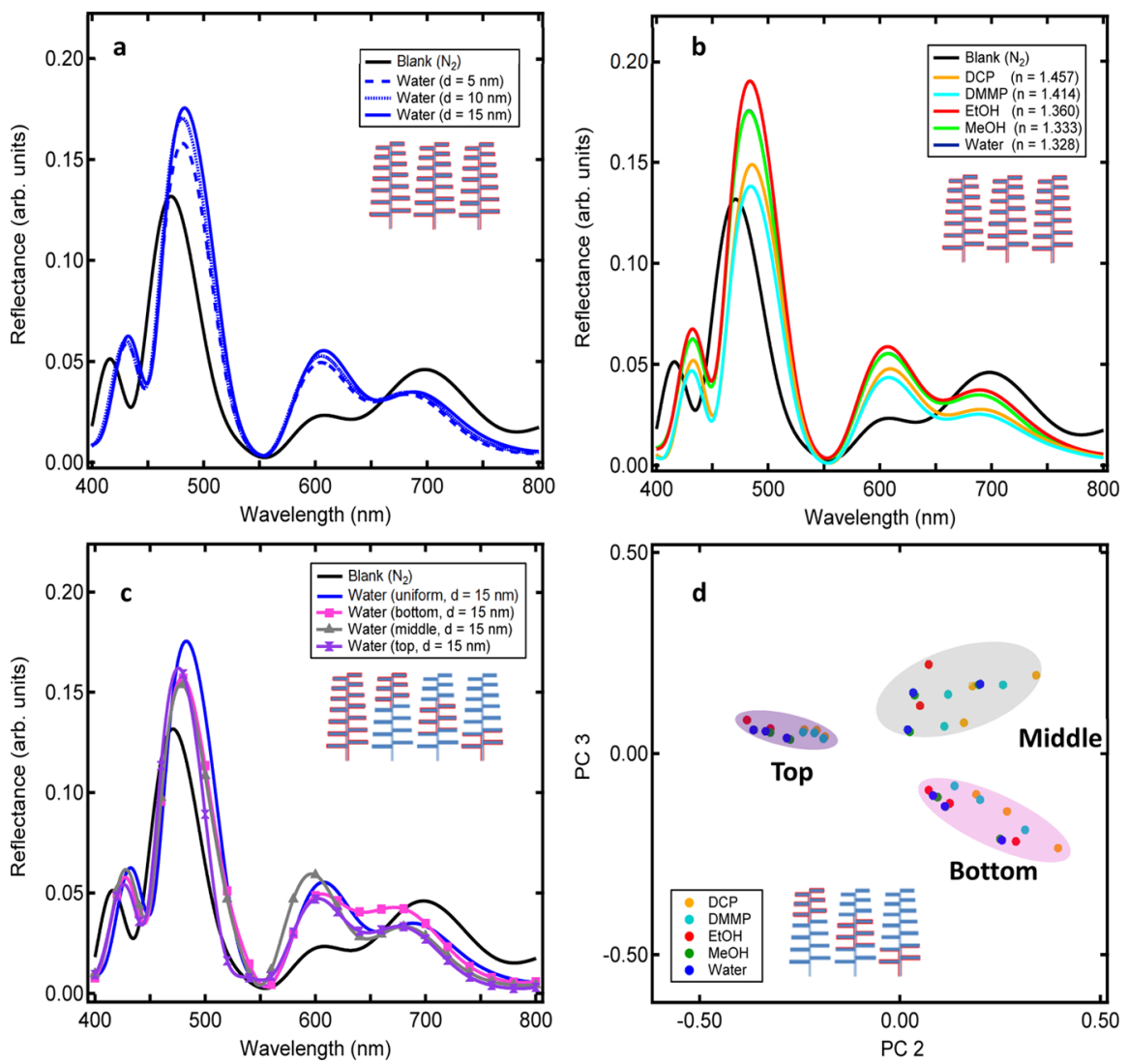

Figure 4. Model reflectance spectra versus wavelength for (a) varied vapor concentration simulated as a film thickness $d$, (b) varied vapor refractive index, and (c) varied vapor location within the lamella. The two-dimensional PCA score plot from Figure S7 of the model $\Delta R$ data (d) highlighting the reflectance variance owing to vapor location within the lamella. Insets show the modeled distribution of vapors within the lamella.

analytes with very similar refractive indices (e.g., water and $\mathrm{MeOH})$, the model yields nearly identical traces $(\mathrm{MeOH}$ trace obscures the water trace), a result not observed experimentally, likely because of the polarity gradient of the natural system and aggregation of analytes in different regions. ${ }^{37}$ Figure $4 \mathrm{~b}$ also illustrates the complex result when the refractive index is varied, as no clear trend is shown in terms of peak intensity or peak shift. For example, EtOH has the highest peak intensity but an intermediate refractive index relative to the other vapors for the model studied. This highlights the interdependent relationships between $\mathrm{PhC}$ structure and spacing, $\mathrm{PhC}$ refractive index and dielectric constant, and analyte refractive index, suggesting that these parameters can be tailored to increase the sensitivity toward an analyte of particular interest. ${ }^{34}$

Similar to other studies, the model data also suggest that the location of a vapor within a $\mathrm{PhC}$ plays an important, even critical, role in the selectivity of natural $\mathrm{PhC}$ systems, especially when considering the combined effects of other parameters, such as concentration and refractive index. Figure $4 \mathrm{c}$ shows the reflectance data when the location of the analyte within the $\mathrm{PhC}$ is varied, whereas the concentration and the refractive index are held constant. Relative to the model with uniform coverage, a blue shift occurred, likely from a decrease in the overall refractive index of the $\mathrm{PhC}$ system. Significantly, differences in the reflectance spectra were observed at most wavelengths for the analyte concentrated in the top, middle, or bottom lamella. A three-dimensional PCA score plot of the modeled $\Delta R$ data for all five vapors is provided in Figure S7. Generally, the data are grouped on the basis of refractive index, concentration, and analyte location within the $\mathrm{PhC}$ structure, with similar values of these variables generating similarities in the traces. A two-dimensional PCA score plot of the first two PCs is provided in Figure S8 and highlights the refractive index and concentration effects.

Of particular interest in this work, the simulated reflectance data for all five analytes concentrated at each of the three locations within the model $\mathrm{PhC}$ demonstrate the variation caused by vapor concentration within certain regions of the model (Figure 4d). A greater variation between analytes was seen in the middle and bottom layers, relative to the top layer, indicating more pronounced spectral distinction between analytes in those layers. This increase in spectral distinction is attributed to changes in spacing between lamellae. For the model used here, a greater vertical separation between lamellae exists in the middle and bottom layers relative to the top layer (Figure S1), suggesting that the increase in vertical separation between lamellae drove the spectral variance. This result is further confirmed by careful examination of work by Potyrailo et al. ${ }^{34,37}$ Although not specifically mentioned by the authors, their modeling data also show increased spectral distinction with increased vertical separation between lamellae. However, in contrast to the work presented here, their model had a greater lamellar separation in the top layer and a corresponding increase in the spectral variance in the top layer over the middle and bottom layers. ${ }^{37}$ Coupled with their result that a tapered lamellar structure was not important for vapor selectivity, ${ }^{34}$ these studies highlight the relevance of lamellar spacing in spectral distinction. Collectively, these results show that targeted functionalization of regions with optimized periodic 
dimensions could lead to increased sensitivity toward a trace analyte.

Results from the modeling suggest several strategies to increase the sensitivity of a synthetic $\mathrm{PhC}$ toward CWA simulants. For example, the refractive index difference between the analyte vapor and the $\mathrm{PhC}$ structure passed through a maximum in the model studied. Thus, selecting a $\mathrm{PhC}$ material with a higher refractive index than chitin should increase the sensitivity toward CWA simulants. Additionally, the lethality of CWA agents is typically expressed in terms of exposure concentration per unit time (e.g., $10 \mathrm{mg} \cdot \mathrm{min} \cdot \mathrm{m}^{-3}$ for VX). ${ }^{47}$ Thus, the functionalization of a PhC surface with a material that binds the CWA of interest (e.g., acetylchlonesterase for VX) could concentrate that analyte with increasing exposure time, resulting in a detectable signal over time that still falls below the lethal exposure dosage. Modeling also showed that spectral differences could be enhanced by altering the periodic spacing of the lamella. Thus, methods for generating polarity gradients or functionalization of the synthetic $\mathrm{PhC}$ mimic should focus on concentrating the analyte vapor in regions of optimized lamellar periodicity. When combined with other known methods to enhance the $\mathrm{PhC}$ sensitivity via operating in stimulated emission regimes, synthesizing higher numbers of lamellae, optimizing ridge spacing, and considering the $\mathrm{PhC}$ extinction coefficient, the detection of CWA simulants should be possible in the parts per billion range or better. ${ }^{34,48}$

\section{CONCLUSIONS}

In summary, this work highlights the first report of sensing CWA simulant vapors via light reflected from a natural $\mathrm{PhC}$. The system was selective toward different simulant vapors and was able to distinguish between analytes of similar polarity and refractive index. Sensitivity toward the analytes was in the parts per million range, although modeling suggested that the sensitivity of a synthetic mimic toward CWA simulants could be improved by the careful selection of $\mathrm{PhC}$ refractive index, targeted functionalization of certain regions within a $\mathrm{PhC}$ nanoarchitecture, and optimized $\mathrm{PhC}$ periodicity. These results are expected to serve as a baseline for future studies of $\mathrm{PhCs}$ as CWA simulant sensors and guide improvements in sensitivity toward these analytes.

\section{MATERIALS AND METHODS}

Materials. Butterflies ( $M$. didius) were purchased from Butterfly Utopia and used as received. DMMP (97\%, D169102) and DCP (99\%, D69602) were purchased from Sigma-Aldrich. EtOH (ACS grade, 111000190) and $\mathrm{MeOH}$ (ACS grade, $339000000)$ were purchased from Pharmco-AAPER. Ultrapure water (Milli-Q gradient A-10, Milli-Q, $18.2 \Omega \mathrm{M} \cdot \mathrm{cm},<5 \mathrm{ppb}$ organic impurities) and ultrahigh purity nitrogen (Airgas, UHP300) were used for all experiments.

Reflectance Measurements. A halogen light source (Ocean Optics, HL-2000) and a spectrophotometer (Ocean Optics, HR2000+) equipped with a fiber optic probe (Ocean Optics, QR400-7-UV-vis) were used for reflectance measurements. The reflectance probe was positioned normal to the butterfly surface, generating an illuminated area about $2 \mathrm{~mm}$ in diameter. In vapor measurements, the differential reflectance spectra $(\Delta R)$ were measured relative to nitrogen. Vapor concentrations were varied using a manual bubbler system to alter the fraction of vapor in the carrier gas so that the partial pressure of the analyte vapor was between $0.15 P_{0}$ and $0.50 P_{0}$, where $P_{0}$ is the saturated vapor pressure at $20{ }^{\circ} \mathrm{C}$. The total gas flow rate for all measurements was $400 \mathrm{~mL} \cdot \mathrm{min}^{-1}$. Each spectrum was collected after $5 \mathrm{~min}$ of exposure to the vapor and processed using binomial smoothing. PCA was performed on the raw spectra after mean-centering the data.

Scanning Electron Microscopy. Butterfly wing samples were mounted to stubs using conducting carbon tape and sputtered with $5 \mathrm{~nm}$ of gold to limit the charge buildup. Scanning electron microscopy images were captured using an FEI Helios NanoLab 600 scanning electron microscope operated at $5.0 \mathrm{kV}$, providing image magnifications up to 30 000.

Optical Modeling. A 2D model based on the ridge and lamella nanostructure of the M. didius wing scales was generated in RSoft DiffractMod (Synopsys), which uses rigorous coupled wave analysis to simulate the diffraction of light moving through an infinite periodic structure. The complex refractive index of chitin with $n=1.56$ and $k=0.06$ was used for the structure. ${ }^{41}$ Various concentrations of the analyte vapor were simulated by coating the structure with a thin film of thickness $d$ and analyte refractive index $n .{ }^{42}$ The incident light was normal to the structure, while the electric field was in-plane.

\section{ASSOCIATED CONTENT}

\section{Supporting Information}

The Supporting Information is available free of charge on the ACS Publications website at DOI: 10.1021/acsomega.7b01680.

Reflectance data, model PhC schematic, and model reflectance data (PDF)

\section{AUTHOR INFORMATION}

\section{Corresponding Author}

*E-mail: joshua.kittle@usafa.edu (J.D.K.).

ORCID $\odot$

Joshua D. Kittle: 0000-0002-7920-2814

Notes

The authors declare no competing financial interest.

\section{ACKNOWLEDGMENTS}

This work was supported by the Defense Threat Reduction Agency (DTRA)-Joint Science and Technology Transfer Officer for Chemical and Biological Defense (MIPR no. HDTRA13964) and the Air Force Office of Scientific Research (AFOSR).

\section{REFERENCES}

(1) Noort, D.; Benschop, H. P.; Black, R. M. Biomonitoring of exposure to chemical warfare agents: a review. Toxicol. Appl. Pharmacol. 2002, 184, 116-126.

(2) Koskela, H. Use of NMR techniques for toxic organophosphorus compound profiling. J. Chromatogr. B: Anal. Technol. Biomed. Life Sci. 2010, 878, 1365-1381.

(3) Pavlov, V.; Xiao, Y.; Willner, I. Inhibition of the acetycholine esterase-stimulated growth of Au nanoparticles: nanotechnology-based sensing of nerve gases. Nano Lett. 2005, 5, 649-653.

(4) DeFrank, J. J.; White, W. E. Phosphofluoridates: biological activity and biodegradation. In The Handbook of Environmental Chemistry; Neilson, A. H., Ed.; Springer: Berlin, 2002; Vol. 3N, p 295.

(5) Costero, A. M.; Parra, M.; Gil, S.; Gotor, R.; Mancini, P. M. E.; Martínez-Máñez, R.; Sancenón, F.; Royo, S. Chromo-Fluorogenic Detection of Nerve-Agent Mimics Using Triggered Cyclization Reactions in Push-Pull Dyes. Chem.-Asian J. 2010, 5, 1573-1585. 
(6) Walker, J. P.; Asher, S. A. Acetylcholinesterase-based organophosphate nerve agent sensing photonic crystal. Anal. Chem. 2005, 77, 1596-1600.

(7) Jenkins, A. L.; Uy, O. M.; Murray, G. M. Polymer based lanthanide luminescent sensors for the detection of nerve agents. Anal. Commun. 1997, 34, 221-224.

(8) Wild, A.; Winter, A.; Hager, M. D.; Schubert, U. S. Fluorometric, water-based sensors for the detection of nerve gas G mimics DMMP, DCP and DCNP. Chem. Commun. 2012, 48, 964-966.

(9) Dale, T. J.; Rebek, J. Fluorescent sensors for organophosphorus nerve agent mimics. J. Am. Chem. Soc. 2006, 128, 4500-4501.

(10) Akthakul, A.; Maklakov, N.; White, J. Improved Vapor Sensitivity by Rationally Designing Fluorescent Turn-on Sensors. Anal. Chem. 2010, 82, 6487-6494.

(11) Jang, Y. J.; Tsay, O. G.; Murale, D. P.; Jeong, J. A.; Segev, A.; Churchill, D. G. Novel and selective detection of Tabun mimics. Chem. Commun. 2014, 50, 7531-7534.

(12) Wu, C.-F.; Cha, H. J.; Valdes, J. J.; Bentley, W. E. GFP-visualized immobilized enzymes: Degradation of paraoxon via organophosphorus hydrolase in a packed column. Biotechnol. Bioeng. 2002, 77, 212-218.

(13) Malosse, L.; Buvat, P.; Adès, D.; Siove, A. Detection of degradation products of chemical warfare agents by highly porous molecularly imprinted microspheres. Analyst 2008, 133, 588-595.

(14) Knapton, D.; Burnworth, M.; Rowan, S. J.; Weder, C. Fluorescent Organometallic Sensors for the Detection of ChemicalWarfare-Agent Mimics. Angew. Chem., Int. Ed. 2006, 45, 5825-5829.

(15) Kanan, S. M.; Tripp, C. P. An infrared study of adsorbed organophosphonates on silica: a prefiltering strategy for the detection of nerve agents on metal oxide sensors. Langmuir 2001, 17, 22132218.

(16) Sohn, H.; Létant, S.; Sailor, M. J.; Trogler, W. C. Detection of fluorophosphonate chemical warfare agents by catalytic hydrolysis with a porous silicon interferometer. J. Am. Chem. Soc. 2000, 122, 53995400.

(17) Adekunle, A. S.; Pillay, J.; Ozoemena, K. I. Electrocatalysis of 2Diethylaminoethanethiol at Nickel Nanoparticle-Electrodecorated Single-Walled Carbon Nanotube Platform: An Adsorption-Controlled Electrode Process. Electroanalysis 2008, 20, 2587-2591.

(18) Kim, Y.; Lee, S.; Choi, H. H.; Noh, J.-S.; Lee, W. Detection of a nerve agent simulant using single-walled carbon nanotube networks: dimethyl-methyl-phosphonate. Nanotechnology 2010, 21, 495501.

(19) Wang, Y.; Yang, Z.; Hou, Z.; Xu, D.; Wei, L.; Kong, E. S.-W.; Zhang, Y. Flexible gas sensors with assembled carbon nanotube thin films for DMMP vapor detection. Sens. Actuators, B 2010, 150, 708714.

(20) Kwon, O. S.; Park, S. J.; Lee, J. S.; Park, E.; Kim, T.; Park, H.-W.; You, S. A.; Yoon, H.; Jang, J. Multidimensional Conducting Polymer Nanotubes for Ultrasensitive Chemical Nerve Agent Sensing. Nano Lett. 2012, 12, 2797-2802.

(21) Kim, H. J.; Lee, J. H.; Lee, H.; Lee, J. H.; Lee, J. H.; Jung, J. H.; Kim, J. S. A Mesoporous, Silica-Immobilized-Nanoparticle Colorimetric Chemosensor for the Detection of Nerve Agents. Adv. Funct. Mater. 2011, 21, 4035-4040.

(22) Yilmaz, M.; Senlik, E.; Biskin, E.; Yavuz, M. S.; Tamer, U.; Demirel, G. Combining 3-D plasmonic gold nanorod arrays with colloidal nanoparticles as a versatile concept for reliable, sensitive, and selective molecular detection by SERS. Phys. Chem. Chem. Phys. 2014, $16,5563-5570$.

(23) Liu, G.; Lin, Y. Electrochemical sensor for organophosphate pesticides and nerve agents using zirconia nanoparticles as selective sorbents. Anal. Chem. 2005, 77, 5894-5901.

(24) Scheide, E. P.; Guilbault, G. G. Piezoelectric detectors for organophosphorus compounds and pesticides. Anal. Chem. 1972, 44, $1764-1768$.

(25) Nieuwenhuizen, M. S.; Harteveld, J. L. N. A surface acoustic wave gas sensor for organophosphorus compounds. Sens. Actuators, $B$ 1994, 19, 502-505.
(26) He, W.; Liu, Z.; Du, X.; Jiang, Y.; Xiao, D. Analytical application of poly\{methyl[3-(2-hydroxy-3,4-difluoro) phenyl] propyl siloxane\} as a QCM coating for DMMP detection. Talanta 2008, 76, 698-702.

(27) Maji, S.; Asrey, R.; Kumar, S.; Saxena, C.; Kumar, N.; Vyas, K. D.; Banerjee, S. Polymer-coated piezoelectric quartz crystal sensor for sensing the nerve agent simulant dimethyl methylphosphonate vapor. J. Appl. Polym. Sci. 2010, 116, 3708-3717.

(28) Guilbault, G. G.; Kramer, D. N.; Cannon, P. L. Electrical Determination of Organophosphorous Compounds. Anal. Chem. 1962, 34, 1437-1439.

(29) Mulchandani, P.; Mulchandani, A.; Kaneva, I.; Chen, W. Biosensor for direct determination of organophosphate nerve agents. 1. Potentiometric enzyme electrode. Biosens. Bioelectron. 1999, 14, 7785.

(30) Choi, N.-J.; Kwak, J.-H.; Lim, Y.-T.; Bahn, T.-H.; Yun, K.-Y.; Kim, J.-C.; Huh, J.-S.; Lee, D.-D. Classification of chemical warfare agents using thick film gas sensor array. Sens. Actuators, B 2005, 108, 298-304.

(31) Jang, Y. G.; Kim, K.; Tsay, O. G.; Atwood, D. A.; Churchill, D. G. Update 1 of: destruction and detection of chemical warfare agents. Chem. Rev. 2015, 115, PR1.

(32) Li, Q.; Zeng, Q.; Shi, L.; Zhang, X.; Zhang, K.-Q. Bio-inspired sensors based on photonic structures of Morpho butterfly wings: a review. J. Mater. Chem. C 2016, 4, 1752-1763.

(33) Xu, H.; Wu, P.; Zhu, C.; Elbaz, A.; Gu, Z. Z. Photonic crystal for gas sensing. J. Mater. Chem. C 2013, 1, 6087-6098.

(34) Potyrailo, R. A.; Bonam, R. K.; Hartley, J. G.; Starkey, T. A.; Vukusic, P.; Vasudev, M.; Bunning, T.; Naik, R. R.; Tang, Z.; Palacios, M. A.; Larsen, M.; Le Tarte, L. A.; Grande, J. C.; Zhong, S.; Deng, T. Towards outperforming conventional sensor arrays with fabricated individual photonic vapour sensors inspired by Morpho butterflies. Nat. Commun. 2015, 6, 7959.

(35) Yablonovitch, E. Photonic band-gap structure. J. Opt. Soc. Am. B 1993, 10, 283-295.

(36) Fenzl, C.; Hirsch, T.; Wolfbeis, O. S. Photonic crystals for chemical sensing and biosensing. Angew. Chem., Int. Ed. 2014, 53, 3318-3335.

(37) Potyrailo, R. A.; Starkey, T. A.; Vukusic, P.; Ghiradella, H.; Vasudev, M.; Bunning, T.; Naik, R. R.; Tang, Z.; Larsen, M.; Deng, T.; Zhong, S.; Palacios, M.; Grande, J. C.; Zorn, G.; Goddard, G.; Zalubovsky, S. Discovery of the surface polarity gradient on iridescent Morpho butterfly scales reveals a mechanism of their selective vapor response. Proc. Natl. Acad. Sci. U.S.A. 2013, 110, 15567-15572.

(38) Potyrailo, R. A.; Ghiradella, H.; Vertiatchikh, A.; Dovidenko, K.; Cournoyer, J. R.; Olson, E. Morpho butterfly wing scales demonstrate highly selective vapour response. Nat. Photonics 2007, 1, 123-128.

(39) Walker, J. P.; Kimble, K. W.; Asher, S. A. Photonic crystal sensor for organophosphate nerve agents utilizing the organophosphorus hydrolase enzyme. Anal. Bioanal. Chem. 2007, 389, 2115.

(40) Niu, S.; Li, B.; Mu, Z.; Yang, M.; Zhang, J.; Han, Z.; Ren, L. Excellent structure-based multifunction of Morpho butterfly wings: a review. J. Bionic Eng. 2015, 12, 170.

(41) Vukusic, P.; Sambles, J. R.; Lawrence, C. R.; Wootton, R. J. Quantified interference and diffraction in single Morpho butterfly scales. Proc. R. Soc. London, Ser. B 1999, 266, 1403.

(42) Yang, H.; Jiang, P. Macroporous photonic crystal-based vapor detectors created by doctor blade coating. Appl. Phys. Lett. 2011, 98, 011104.

(43) Jiang, T.; Peng, Z.; Wu, W.; Shi, T.; Liao, G. Gas sensing using hierarchical micro/nanostructures of Morpho butterfly scales. Sens. Actuators, A 2014, 213, 63.

(44) Goll, E. S.; Jurs, P. C. Prediction of vapor pressures of hydrocarbons and halohydrocarbons from molecular structure with a computational neural network model. J. Chem. Inf. Comput. Sci. 1999, 39, 1081 .

(45) Butrow, A. B.; Buchanan, J. H.; Tevault, D. E. Vapor pressure of organophosphorus nerve agent simulant compounds. J. Chem. Eng. Data 2009, 54, 1876. 
(46) Abdi, H.; Williams, L. J. Principal component analysis. Wiley Interdiscip. Rev. Comput. Stat. 2010, 2, 433.

(47) Sidell, F. R. Nerve Agents. In Medical Aspects of Chemical Biological Warfare; Zajtchuk, R., Bellamy, R. F., Eds.; Office of the Surgeon General: Bethesda, 1997; p 141.

(48) Noh, H.; Yang, J.-K.; Liew, S. F.; Rooks, M. J.; Solomon, G. S.; Cao, H. Control of lasing in biomimetic structures with short-range order. Phys. Rev. Lett. 2011, 106, 183901. 